\title{
Hydrodynamics Near the Central Engine
}

\author{
John F. Hawley
}

Theoretical Astrophysics 130-33, Caltech Pasadena, CA 91125

\begin{abstract}
The "central engine" of quasars and active galactic nuclei is most likely powered by accretion into a supermassive black hole. A menagerie of steady state accretion flows have been developed, and these are briefly reviewed. Several examples from recent numerical calculations of axisymmetric accretion flows provide disk formation scenarios from which one can calculate plasma densities, and other values of interest for a range of accretion rates and black hole masses. These results can be compared with the well studied, but physically unrealistic case of radial accretion. Although a reasonable conceptual picture of the central engine has been developed, considerable work remains to be done.
\end{abstract}

\section{Introduction}

Hith the advent of the radio telescope in the early 1960's, and the more recent development of radio interferometry, it has become apparent that the universe contains a wide assortment of highly energetic phenomena. These include the radio loud and quiet quasars, Seyfert galaxies, radio galaxies, and BL Lac objects. Such "active galactic nuclei" (AGN) are typically characterized by a compact nonthermal radio source and extended radio lobes, now believed to be associated with jets beamed from the region of the compact source. These objects have presented us with a major theoretical problem: what are the central engines which power these sources. and how do these engines work?

A great difficulty in developing a theory of central engines is the lack of direct observations of the cores of AGNs. We can only infer general properties of the engine through observations of the surrounding galaxies, emitting regions, and jets. However, the growing body of observational evidence points to several important conclusions: the central engine is very compact, it can produce copious amounts of energy, both in nonthermal continuum and relativistic outflows, and, in the case of sources associated with radio jets, it maintains a preferred directionality over a significant time.

It is just such circumstantial observational evidence which has led investigators to suspect that black holes lurk at the heart of AGNs. Typically, energies in quasars are on the order of the Eddington luminosity for a $10^{8} \mathrm{M}_{\odot}$ black hole (10 $0^{46}\left[\right.$ ergse $\left.\left.^{-1}\right]\right)$ so accretion onto sufficiently massive holes could in principle provide the requisite energy. Such a luminosity is equal to $0.23 M_{\odot}$ per year which implies that over the quasar lifetime, substantial mass (on the order of $10^{8} M_{\odot}$ ) must have been processed in the central engine. While there are other possible quasar engines (stellar clusters, supermassive stars), they must also meet these fundamental observational requirements of energetics and compactness. The "inevitability of collapse" for a large mass in a compact region is noted by REES [1], who points out that the evolutionary endpoints for these more "conventional" central engines are most likely massive black holes. A slightly more speculative point is that the spin axis of a black hole provides an appealingly stable bi-directionality for those concerned with jet production. Also one suspects that a relativistic engine is required to produce the relativistic kinematics observed in some jets.

Despite considerable gaps in both theory and observation, ideas such as these have been used to form a reasonable working picture of the central engine. It is powered by the dissipation 
of gravitational binding energy in accreting plasma. Alternately, or in addition, the spin energy of the hole itself may provide a significant power source. By way of $L_{E d d}$ the mass of the hole provides the fundamental scale for the central engine luminosity. The fraction of $L_{B d d}$ actually generated will depend on the accretion rate, scaled in the same way by the hole's mass. These ideas suggest that the wide variety of observed energies in both the jets (if present), and compact sources in AGN is to lowest order produced by variations in the mass of the black hole and the accretion rate (the central engine's H-R diagram).

Although armed with motive, opportunity, and good alibis for the other major suspects, a conviction requires a detailed description of the black hole's modus operandi. This is a challenging task since the black hole accretion mechanism must certainly involve complex physical processes such as relativistic MHD with dynamically important radiation. Kinematic velocities may be on the order of the speed of light, and the energy density in the radiation field may be comparable to $m_{p} c^{2}$. Thus we are dealing with what $\mathrm{Dr}$. Mihalas in his opening talk referred to as an "Exotic Phenomenon", i.e. not well understood.

\section{Qualities of Black Holes}

Compared to the accretion disk which surrounds it, the black hole is a relatively simple object. It is parameterized by its mass $M$, and specific angular momentum $a(<1)$. The hole's simplicity allows a oomplete description of the accretion system in terms of the black hole's mass. The first black hole unit is that of distance, namely the "gravitational radius",

$$
r_{0}=G M / \mathrm{c}^{2}=1.5 \times 10^{5} \mathrm{M} / \mathrm{M}_{\odot}[\mathrm{cm}] .
$$

The horizon of a Schwarzschild $(a=0)$ black hole is $2 r_{g}$. The unit of time is

$$
r_{0} / c=5.0 \times 10^{-6} M / M_{\odot}[\text { sec }] \text {. }
$$

The canonical luminosity for the black hole is the Eddington luminosity for an object of mass $M$,

$$
L_{\text {Bdd }}=1.3 \times 10^{38} \mathrm{M} / M_{\odot}\left[\mathrm{erg} \mathrm{sec}^{-1}\right] \text {. }
$$

The associated accretion rate is

$$
\dot{\mathrm{H}}_{\mathrm{E}}=L_{\mathrm{Edd}} / \mathrm{c}^{2}=1.4 \times 10^{17} \mathrm{M} / \mathrm{M}_{\odot}\left[\mathrm{gm} \mathrm{sec} \mathrm{se}^{-1}\right] \text {. }
$$

For a $10^{8} M_{\odot}$ black hole these values are $r_{g}=1 \mathrm{~A} . U ., t=500 \mathrm{sec}, L_{B d d}=1.3 \times 10^{48} \mathrm{erg} / \mathrm{sec}$, and $\dot{H}_{E}=0.23 M_{\odot} y r^{-1}$. Since all these physical values scale directly with the black hole mass, calculations in terms of these parameters are scale free and can be applied to both quasars and stellar mass objects such as Cyg X-1. A considerable amount of the work done to date derives directly from these basic black hole scaling laws.

As a benchmark example, consider the values obtained in an idealized radial inflow. Assume the inflow velocity is some fraction $\alpha$ of the freefall velocity

$$
v_{i n a l l}=\alpha v_{\tilde{J}}=\alpha(2 / r)^{k}
$$

where $r$ is in units of the gravitational radius. The value $\alpha$ will be reduced below one by pressure in the fluid, by angular momentum, etc. The density associated with such an infall is given by

$$
\rho=2 \times 10^{-5} \alpha r^{-3 / 2}\left(M / \dot{M}_{E}\right)\left(M_{\odot} M\right)
$$

with the accretion rate scaled by the Eddington rate. The optical depth to Thomson scattering in such a flow is approximately

$$
\tau_{T} \approx\left(M / \dot{M}_{E)} \alpha^{-1}(r / 2)^{-1 / R} .\right.
$$

Thus optical depth can be large for large accretion rates or for small inflow velocities $(\alpha \ll 1)$.

There are several properties which make accretion onto black holes unique as compared with Newtonian accretion. First, a black hole has no solid surface, and can swallow arbitrarily large amounts of matter and radiation. Thus there is no set accretion efficiency such as can be associated with the surface binding energy of a neutron star or white dwart. The net efficiency in black hole accretion depends on two factors: the ability of the infalling fluid to dissipate gravitational binding energy as it infalls, and the fraction of that released energy to escape the hole. 
A second relativistic effect has to do with the nature of Keplerian orbits around a black hole. The "effective potential" is a familiar cbncept from Newtonian orbital mechanics, where the presence of angular momentum creates a potential barrier isolating the central point mass, and determining the turning point in orbits of different energies. Around a black hole however, the effective potential does not rise to infinity near the black hole; gravitational attraction dominates at small radius. There is a minimum circular orbit, and an associated minimum angular momentum, the marginally stable value $l_{m s}$, where $l=-U_{\varphi} / U_{t}$, and $U_{\alpha}$ is a covariant component of the four velocity. In Schwarzschild geometry, this orbit is located at $r_{m s}=6 M$. A second fiducial angular momentum is the "marginally bound" value, $l_{m b}>l_{m s}$. This is the minimum angular momentum which will provide a turning point for a marginally bound particle, i.e. one falling from rest at infinity. (See [2] for a more complete discussion of orbits around black holes.)

We can use the effective potentials, and their angular momenta to describe expected behavior of particles in orbit around a black hole. For example, fluid with angular momentum less than $l_{m s}$ can fall into the hole without any further aid from dissipative torques. Thus, the marginally stable orbit serves as an estimate for the net accretion efficiency: dissipation continues down to $r_{\text {ms }}$ inside of which the fluid freefalls into the hole. Similarly, fluid with angular momentum $l \geq l_{m b}$ will encounter the centrifugal barrier and not enter the hole until its angular momentum is reduced by some means.

The full two-dimensional equipotential surfaces provide one means of describing the surface of orbiting accretion tori [3],[4]. One such equipotential surface is the centrifugal barrier which defines the accretion funnel, a centrifugally evacuated vortex along the fluid's orbital axis. What differentiates the relativistic funnel from a similar Newtonian vortex is the presence of a hole at the bottom of the funnel which insures that fluid inside the funnel cannot be static. It was this property that led to the development of the idea that jets might be formed by radiatively accelerating material in the funnel outwards while collimating the beam with a surrounding accretion torus [5]. Some problems with and variations on this idea are discussed by Blandford in this volume.

A third important relativistic phenomenon is the dragging of inertial frames associated with spinning (Kerr) black holes. It has been suggested that this can enforce axisymmetry near the hole in an accretion flow [6] by creating torques which bring the fluid's orbital axis into line with the spin axis of the hole. A spinning black hole also offers an alternate energy source to the usual dissipation in the accreting fluid. The energy which can be extracted from a maximally rotating black hole can be considerable in principle, up to $29 \%$ of the rest mass of the hole [2]. A variety of mechanisms have been proposed which could extract some of that energy (e.g. [7]. [B]).

\section{Accretion Scenarios}

Before exhibiting some computational models, I will briefly review some general properties of various accretion scenarios that have been investigated. The emphasis will be on those aspects unique to black holes, and the radiative properties of the models. Additional details can be found in recent review articles [9],[10], and references therein.

Researchers have mainly divided their attention between (approximately) steady-state disk models, and spherically symmetric accretion. The later simplification makes possible the inclusion of many physical effects including radiation transport in some limits. In the ideal hydrodynamic limit the sole source of heating is $P d V$ work as the gas accretes. SHAPIRO [11] has calculated models with optically thin emission and shown that, typically, such flows are very inefficient. For some large effective adiabatic $\Gamma$ models, such as one by MESZAROS [12] invoking magnetic turbulent heating, or models with optically thick inflows [13], [14], substantially increased efficiency is possible. However, assuming that the dominant opacity is Thomson scattering, by the time an Eddington luminosity has been generated, the inflow velocity exceeds the outward diffusion velocity for the radiation, and most of the heat generated is swept into the hole [15]. Thus the lack of a hard surface in a black hole acts to reduce the net radiation emerging from a spherical inflow. This conclusion is supported by the time-dependent numerical calculation by GILDEN and WHEELER [16], and by the analytic calculations of BEGELMAN [13], and FLAMMANG [14]. Although the radiation apparently cannot transfer sufficient momentum to the infalling fluid to halt spherical accretion, OSTRIKER et. al. [17] suggest that emerging radiation 
may transfer energy to the infalling fluid at large radius, preheating the fluid and shutting off inflow. Time-dependeńt, one-dimensional calculations by COWIE, OSTRIKER, and STARK [1B] have generated such oscillatory flows.

The spherically symmetric studies raise several questions, particularly regarding the small efficiency of accretion into black holes, and the manner in which the resultant luminosity can be radiated to infinity. Of fundamental importance is the geometry of the inflow which will depend in large part on the angular momentum in the fluid. While no flow is expected to have identically zero angular momentum, how low must $l$ be to be insignificant? What are the realistic inflow geometries? Do these geometries provide optically thin escape routes for radiation, or alter the "preheating" of infalling fluid? These and other similar issues can be dealt with only by solving the full multi-dimensional, time-dependent equations.

Accretion models in which angular momentum plays the dominant role are disk models. There are essentially two classes of disks: thin disks and tori, Thin disks [19], [20] are confined to Keplerian orbits in the equatorial plane. Viscous torques between differentially rotating rings of the disk provide the means to generate heat and transport angular momentum. Prompt cooling by radiation emission prevents the development of vertical thickness in the disk. The black hole models resemble the disk models proposed for white dwarfs and neutron stars (see [21] and articles by Meyer and by Bath in this volume.) The one major difference occurs at the inner boundary of the disk where a stellar surface is replaced by the innermost (marginally stable) Keplerian orbit. Inside of this orbit the gas flow proceeds dynamically into the hole, probably without further significant heat gain or loss. The energies associated with these orbits yield efficiencies ranging from about 6 to up to $42 \%$ for a maximally rotating Kerr hole [20]

The fraction of the generated radiation which is swallowed by the hole is uncertain, but since the radial drift velocity in the disk is likely to be much less than the freefall value, such losses will be less than in spherical accretion. Loss down the hole will be most significant in Kerr hole cases where the inner edge of the disk is very close to the horizon. The total luminosity emerging from the thin disk is usually regarded to be constrained by the Eddington limit. Some possible consequences of super-Eddington accretion include disruption of the disk, enhanced losses down the hole, and the development of a significant radiation-driven wind [19].

Tori are disk models in which heat generation in the disk exceeds the loss from radiation. If the gas is optically thick, radiation pressure can provide the internal pressure support, creating an extended vertical structure in the disk. Investigations of these disks have centered on their global properties as determined by the effective gravitational potential around the hole [22]. The surface of the disk, where density and pressure drop to zero, radiates at the local Eddington value. Such circumstances are very likely to drive a wind from the disk, including a wind into the funnel. The intensity of the radiation in the funnel appealed to investigators as a possible jet acceleration mechanism [23], [24], but the radiation is relatively isotropic deep in the funnel, and does not accelerate optically thin material efficiently [25]. Optically thick acceleration seems to be more promising [26]. Such a flow might be generated by strong local energy release near the hole, or by a vigorous wind injecting substantial matter into the funnel.

A related entity is the cauldron model of BEGELMAN and REES [27]. In this picture, superEddington accretion creates a geometrically and optically thick region of matter surrounding the hole. Strong local heating due to shocks or MHD processes release substantial amounts of energy in this near hole region. The super-Eddington luminosities thus generated create substantial mass and radiation outflow along the orbital axis.

Note that the requirement that the radiation torus be optically thick means that either the accretion rate must be large $\dot{M} / \dot{M}_{E}>1$, or the inflow rate very small, $\alpha \ll 1$. If the disk is optically thin, the electrons will promptly cool by inverse Compton or synchrotron emission. If the timescale for ion to electron coupling is sufficiently long. a two temperature torus develops. Such models have been proposed to explain the non-thermal X-ray emission from Cyg X-1 [28]. [29]. REES et. al. [8] have suggested that such ion-supported tori may support magnetic fields which extract spin energy from the black hole. This mechanism could power radio jets in AGN which have no detectable central emission, and hence cannot contain tori radiating at $L_{B d d}$. Whether or not these two temperature tori can exist depends critically on the physics of high 
temperature, extremely low density plasma. Since this physics is not presently understood, these models remain speculative.

\section{Constant Angular Momentum Inflows}

The one-dimensional radial accretion problem is analytically tractable, but not realistic. The addition of angular momentum creates flows which are inherently multi-dimensional. Such flows can be studied by using simplifying assumptions (steady state, flow confined to equator), or by solving the full multi-dimensional equations numerically. The first black hole accretion code was written by WILSON [30]. His approach of finite differencing the equations of general relativistic ideal fluid dynamics has recently been updated and calibrated by HAWLEY, SMARR, and WILSON [31], [32]. This paper reports on some results obtained from using this code to supplement the developing central engine picture with insights into the hydrodynamic behavior of gas near a black hole.

Perhaps the simplest extension to the spherically symmetric inflow problem is to add a fixed quantity of angular momentum to the infalling fluid. Since the centrifugal acceleration diverges at the orbital axis, such a flow represents a singular deviation from the spherically symmetric case as one approaches the axis. In other words, this flow is guaranteed to produce an evacuated funnel. In addition, from an analysis of test particle orbits, it is clear that particles with angular momentum $l<l_{m s}$ can enter the black hole, while those with $l>l_{m b}$ are shielded from the hole by a centrifugal barrier.

Thus armed with expectations derived from test particles, we approach the more complicated fuld dynamical case. This discussion will be presented in terms of one particular calculation, namely that of a fuid with angular momentum $l_{m s}<l=3.8<l_{m b}$ accreting into a Schwarzschild hole. Such a fluid has a circular orbit at $r_{\text {kep }}=8$, an orbital time at this radius of $t_{\text {kop }}=140 \mathrm{M}$, and a freefall time from the outer edge of the grid $(100 \mathrm{M})$ of $t_{f \sigma}=500 \mathrm{M}$. At this Keplerian orbit, the binding energy is $0.051 \mathrm{mc}^{2}$. This model will be used to illustrate the flow patterns that result in constant angular momentum inflows. Additional calculations are presented in [33].

In all the models to be described here, the numerical grid is differenced into 160 equally spaced zones from 0 to $\pi$ in $\theta$, and 160 logarithmically space radial zones from one to 50 horizon radii (in Schwarzschild geometry from 2 to $100 \mathrm{M}$ ). The fluid is sent onto the grid over a wide angle up to where the funnel wall intersects the outer boundary. The fluid is bound (binding energy $1+U_{t}=0.007$ ), has been given a purely radial inflow velocity, and it is initially cold and hence supersonic at the outer boundary (mach 10). The equation of state is taken to be an ideal gas with $\Gamma=4 / 3$.

At the beginning of the example calculation, fluid with angular momentum $l=3.80$ flows radially onto the grid. Soon, however, a non-radial velocity component builds as the fluid begins to accelerate towards the equator. The higher the fluid above the equatorial plane, the greater this acceleration. The flow quickly develops a $\theta$ component of velocity which is supersonic with respect to the internal sound speed of the gas. The high latitude fluid trajectories intersect with those at lower latitudes producing a "funnel wall standoff shock" along the surface of the inflow. Inside this shock the fluid is turned sharply towards a convergence point located at $r=19 \mathrm{M}$ on the equator. Here, shocks redirect the fluid towards the hole, and the now quite hot fluid expands laterally as it flows inward. The compression and shocks at the convergence point on the equator produce a fluid internal energy about 50 times greater than produced by PdV work in the equivalent spherical inflow. Figure One shows the flow patterns in this critical near hole region at $t=720 \mathrm{M}$, slightly in excess of one freefall time.

The subsequent fate of the gas depends on its angular momentum. For $l<l_{m s}$ there is no circular orbit, and the effective potential does not turn up to create a centrifugal barrier. An example of such an inflow is given by the accretion of $l=3.0$ fluid. During the initial infall phase, this low $l$ flow closely resembles the $l=3.80$ case described above. As before, the fluid forms funnel wall shocks as it is focused toward the equator. These shocks cross at the equator where the fluid is redirected inwards to the hole in exactly the same manner as for the higher $l$ case. A steady state configuration quickly develops which features an evacuated funnel, and heating in 
excess of the spherical case.

However, in flows with greater angular momentum, such as the $l=3.80$ model, circular orbits and orbital turning points are possible. The resulting centrifugal deceleration produces an accretion shock which forms at $r=r_{k \in p}=8 \mathrm{M}$. Behind this shock the accreting fuid has been brought essentially to rest. A hot, thick disk begins to grow as the accretion shock slowly backs out of the potential well

The post-shock, high pressure fluid will try to expand to fill the region of space available to it as defined by the effective potentials. One place the high pressure will force the fluid is inward to the orbit of zero binding energy (at $r=4 M$ for a Schwarzschild hole). In fact, if the angular momentum in the fluid is less than the marginally bound value it can be pressure driven from the torus and into the hole over the cusp in the total effective potential (the "hole in the funnel wall"; see [4]). Since the hole swallows material with angular momentum $l>l_{m s}$ from an orbit which is less tightly bound than marginally stable, the net accretion efficiency is lower than in the thin disk case. Such an accretion "nozzle" can be seen in Fig. 1. The flow through this nozzle is transonic with a sonic point located interior to the unstable circular orbit. In this example calculation, inflow into the hole through this nozzle continues throughout the evolution, although the inflow rate is only $4 \%$ of that coming in at the outer boundary. It may not be possible to maintain a nearly constant angular momentum "nozzle" such as this when viscosity is present. Any torque will tend to reduce the angular momentum, opening up the nozzle, and increasing inflow.

This calculation demonstrates the multiple sonic point nature of accretion flows into black holes. As anticipated by ABRAMOWICZ and ZUREK [34], flows with low $l$ have sonic points at large radius (quasi-spherical fows), while higher $l$ (disk) flows have sonic points near the hole in a confined equatorial inflow stream. ABRAMOWICZ and ZUREK postulated that the transition between these two states would be discontinuous, a situation they refer to as rotation-induced bistability. Here we see just such a discontinuous transition in the form of the accretion shock and disk formation.

Figure 1 illustrates the second route that the pressure-driven fluid takes. This is back out away from the hole and the funnel wall. The surface of the inflow provides inertial confinement for the backflowing fluid, and it is forced up along an empty "escape route" between the inflow surface and the funnel wall. This creates a "hollow jet" of fluid driven from the near hole region.

As the evolution continues, the features created in the initial infall persist in the absence of viscosity. In Fig. Two a late time density contour plot has been labeled to show the locations of relevant structures. Note how effectively the fluid is excluded from the funnel. The labeled funnel wall shock and the accretion shock both move out through the grid so the calculation never reaches a steady state.

The thick disk which has been formed by the shock heating of infalling constant $l$ fluid resembles the analytic constant $l$ thick disk [4] insofar as its shape, like the analytic disk, is primarily determined by the shape of the black hole's effective potential. Figure 3 compares the internal energy along an equatorial slice in this thick disk with that calculated for an equivalent analytic disk in which all the gravitational binding energy is converted in thermal energy. In the analytic disk, the interior is isentropic, and has no internal motion. Owing to its genesis in a series of shocks, the model disk has a multientropic, circulating interior.

The numerical calculations provide some self-consistent information about the structure in the disk. Properties of this disk can be compared with estimates made from spherical accretion. From (6), the density at $r_{k 8 p}=8 M$ in a spherical inflow is

$$
\rho=7.5 \times 10^{-7}\left(M / \dot{M}_{E}\right)\left(M_{\circlearrowleft} / M\right)
$$

In the model disk at the pressure maximum (located at $r_{k e p}$ ) $\rho_{\max }=4 \times 10^{-5}\left[\mathrm{gm} \mathrm{cm}^{-9}\right]$, times the same factors for accretion rate and hole mass. Similarly, the average density in the post shock torus is $10^{-6}$. The calculated torus volume is about that of a sphere of radius $65 \mathrm{M}$. In a freefall spherical accretion flow, the average density in such a sphere would be $3 \times 10^{-8}$, so the torus is roughly 30 times the density of an equivalent radial flow. The optical depth is also up, but due to the limited physical extent of the torus, $\tau$ is increased only by a factor of 4 . As in the spherical accretion case, optically thick flows result from accretion rates in excess of $\dot{M}_{E}$. 


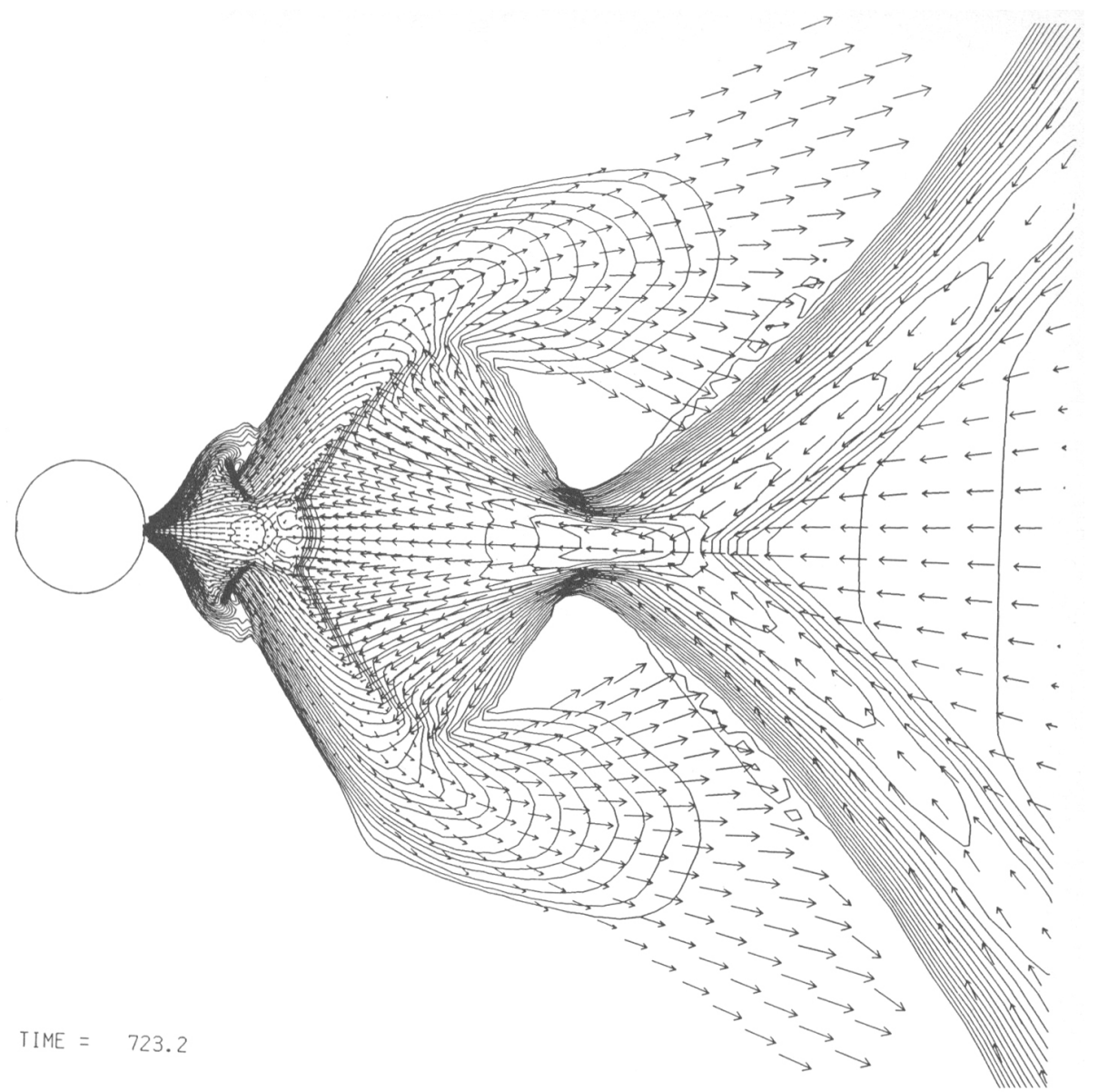

Figure 1: Closeup of the $l=3.80$ accretion flow showing flow convergence to the equator, formation of the accretion shock, nozzle inflow into the hole, and the fluid "splash back" from the funnel wall

Given the high temperatures in the thick disk (Fig. 3) it is clear that the electrons will cool on a timescale much shorter than the dynamic disk formation timescale. If this radiation is promptly lost from the disk, the disk's evolution will depend significantly upon the coupling timescale between the protons and electrons. Thus, for moderate accretion rates on order $M_{E}$ or less, the disk will most resemble the ion torus described above.

Similar inflow models have been computed using a near maximal Kerr hole $(a=0.98)$. The Kerr geometry made little difference beyond modifying the shape of the total potential curves, locations of circular orbits, and related quantities. In a pure hydrodynamic flow there is no way to couple the near hole fluid which could be affected by the rotation of the hole, with the supersonically infalling fuid at larger radius. The inclusion of radiation transport, and/or magnetic fields, would allow significant new effects, such as the extraction of spin energy from the hole. 


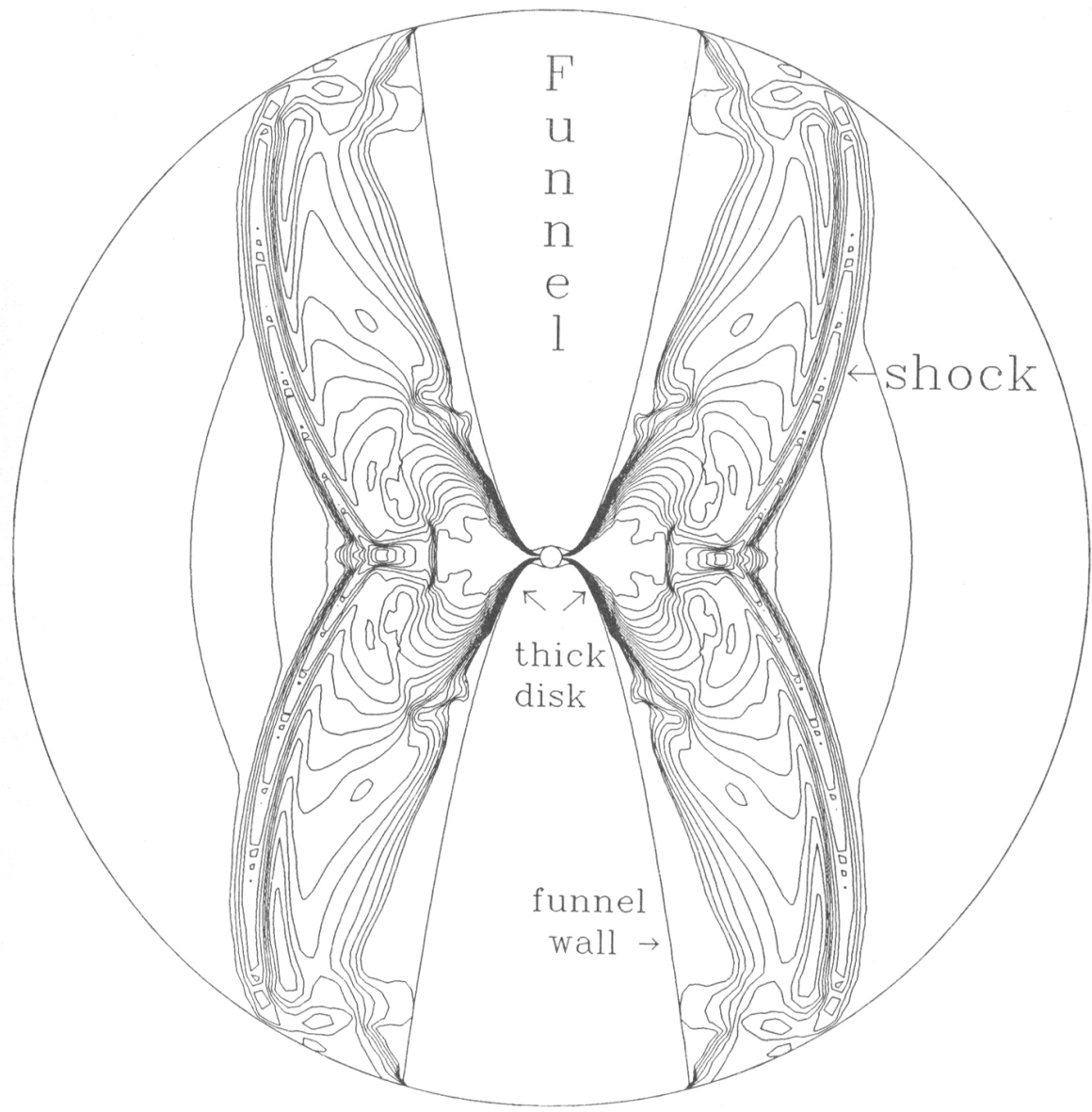

Figure 2: Late time density contour plot showing the shock structures, thick disk, funnel, and hollow jet for the $l=3.80$ inflow model

\section{Accretion of Fluid with an Angular Momentum Gradient}

The fact that the centrifugal acceleration diverges near the orbital axis means that constant angular momentum inflows are essentially singular deviations from the spherical accretion case; a funnel must always form. To avoid this necessity, consider instead the inflow of fuid with a distribution of angular momentum proportional to $\sin ^{2}(\theta)$, i.e. the fluid has constant angular velocity at the outer boundary. In the model described here, fluid is sent onto the grid with angular momentum $l=9 \sin ^{2}(\theta)$. Integrating along the outer boundary reveals that $25 \%$ of the infalling fluid will have $l<l_{m s}$. The average value of $l$ for this portion of the fluid is 2.1 , whereas the average value of $l$ for the high angular momentum portion of the fluid is 7.1 . If the flow is laminar we anticipate that the low $l$ fluid will enter the hole while the high $l$ fluid will encounter an orbital turning point, and form a disk. The explicit numerical calculation tests these expectations.

A 160 by 160 grid identical to the one used above for the constant $l$ models is again employed, and the inflowing fluid is highly supersonic. The inflow velocity at the outer boundary $(100 \mathrm{M})$ is the radial freefall value appropriate to the angular momentum at each angle. This is given by equation (5) with $\alpha=k /\left(4 r_{b}\right) l$ (here $k=9$ ). 


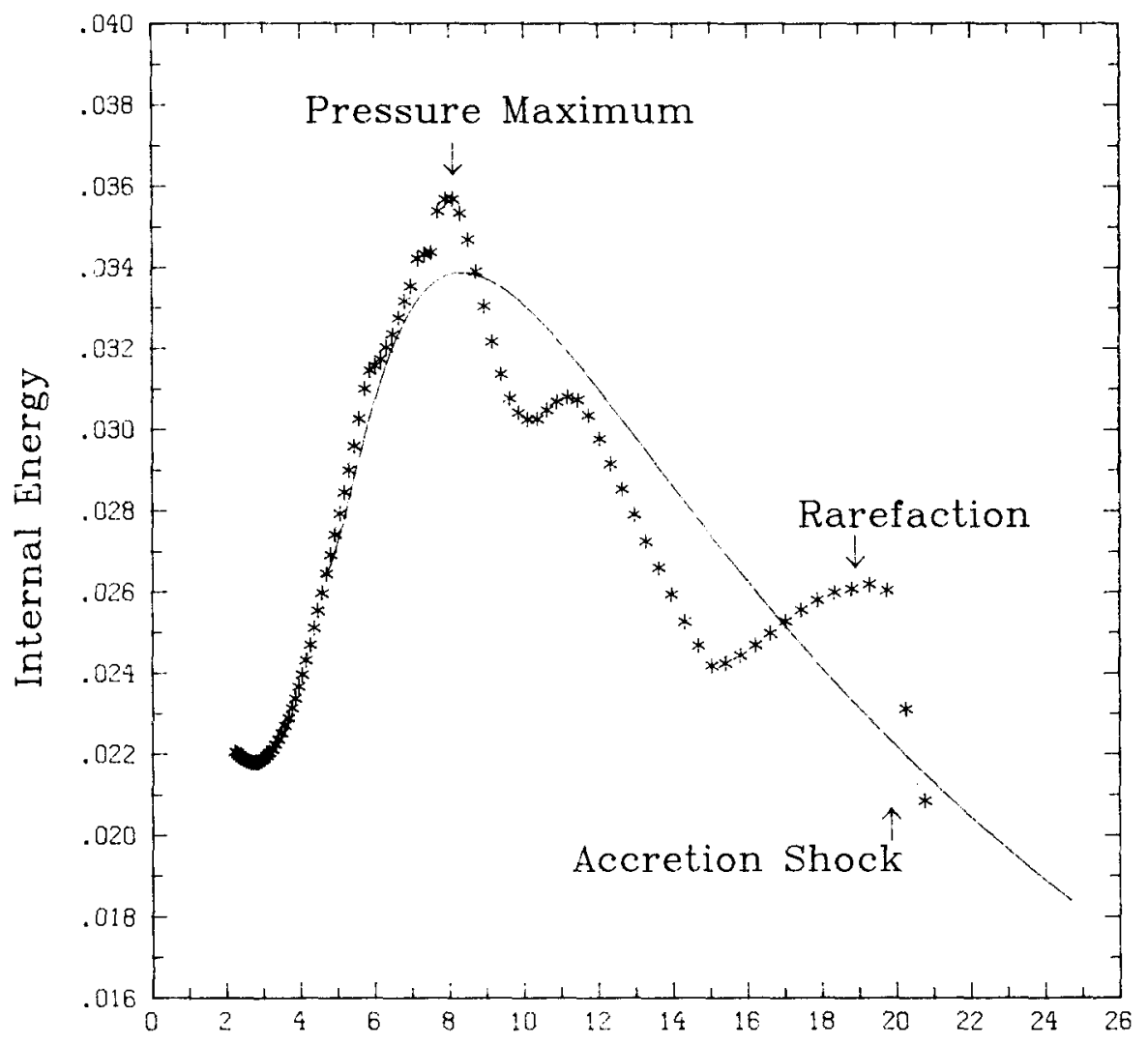

radius

Figure 3: A comparison of the numerically calculated internal energy along the equator (*) with the internal energy the analytic thick disk (solid line); the location of several features in the inflow is indicated

As the inflow begins, the fluid acquires a non-radial velocity component. Figure 4 depicts lines of constant angular momentum which are essentially flow lines in the fluid. Each flow line intersects the equator at a point inside of its Keplerian orbit. This creates a shock along an extended line from the horizon out to $27 \mathrm{M}$. As fluid continues to rain down on the equator, the shocked region grows. The fluid with angular momentum less than $l_{m s}$ is turned at the shock so that it flows into the hole along the equator. Over the course of the evolution, this low $l$ fluid is able to establish a steady state. On the other hand, material with $\iota>l_{m s}$ does not go into the hole. Rather it bounces back along the equator in a confined stream, inflating a hot torus of fluid. As the growing torus encounters the continuing inflow, shocks form along the torus surface. The accretion disk grows both from fluid which has shocked deep in the potential and been ejected along the equator, and from fluid which is shocked at the torus surface, descends towards the equator, and joins the equatorial outflow in a large circulation pattern. The late time structure of the disk is displayed in Fig. Five in both density and pressure,

The mass in the disk grows linearly with time throughout the calculation. As expected from the analysis of the inflow boundary conditions, $74 \%$ of the mass which enters the grid, that portion with $l>l_{\text {ms }}$ ends up in the torus. The low $l$ remainder goes down the hole. The lower the angular momentum, the deeper in the potential it shocks. Thus, the hottest fluid in the disk is 


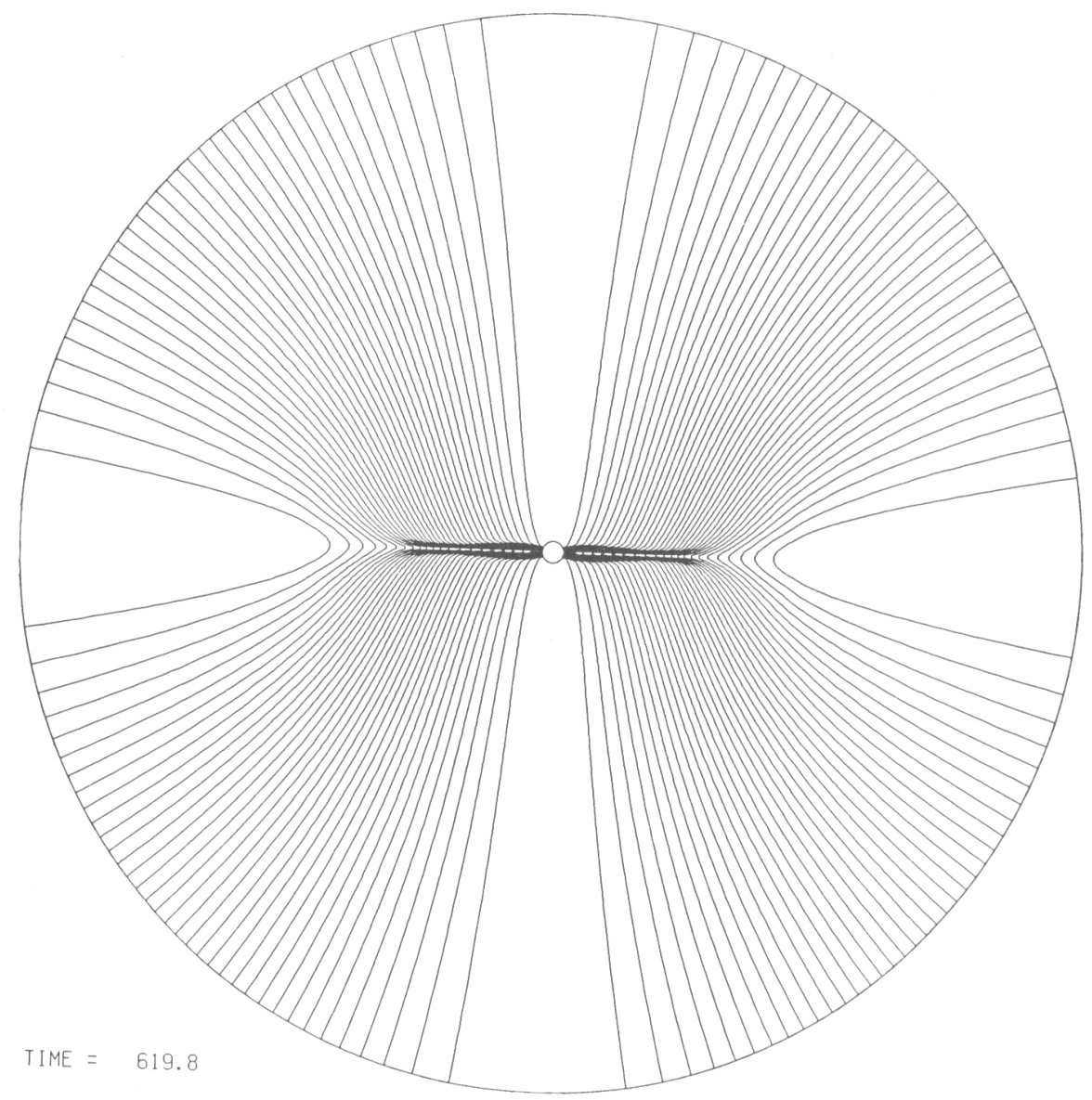

Figure 4: Contours of constant angular momentum in the initial inflow of the $l=9 \sin ^{2}(\theta)$ model. Intersecting fluid lines create a shock along the equator

the low $l \approx l_{m s}$ fluid which flows out along the equator. As in the constant $l$ models described above, the torus which is formed is not in internal equilibrium. Strong gradients in fluid variables drive large scale circulations.

As before, fluid properties in this disk can be compared with the radial accretion flow. The average internal energy in the disk is $\varepsilon_{a v g} \approx 10^{-2}$, approximately the same as the binding energy of the Keplerian orbit at $40 \mathrm{M}$. The average density is $\rho_{\text {aug }}=9 \times 10^{-7}$, about 10 times the freefall density at that same Keplerian orbit. The optical depth is twice the total optical depth for the equivalent radial infall problem. As in the constant angular momentum case, the formation of an optically thick torus requires a super-Eddington accretion rate.

\section{Concluding Questions}

While these numerical calculations indicate that thick tori can form around black holes, they give no indication as to the subsequent evolution of such tori. Several important effects are clearly radiation dominated, and they have not yet been investigated in sufficient detail. As mentioned above, the radiative properties of optically thin, relativistically hot torus are very uncertain. Do two temperature tori exist, and what is their effective ion-electron coupling timescale? 


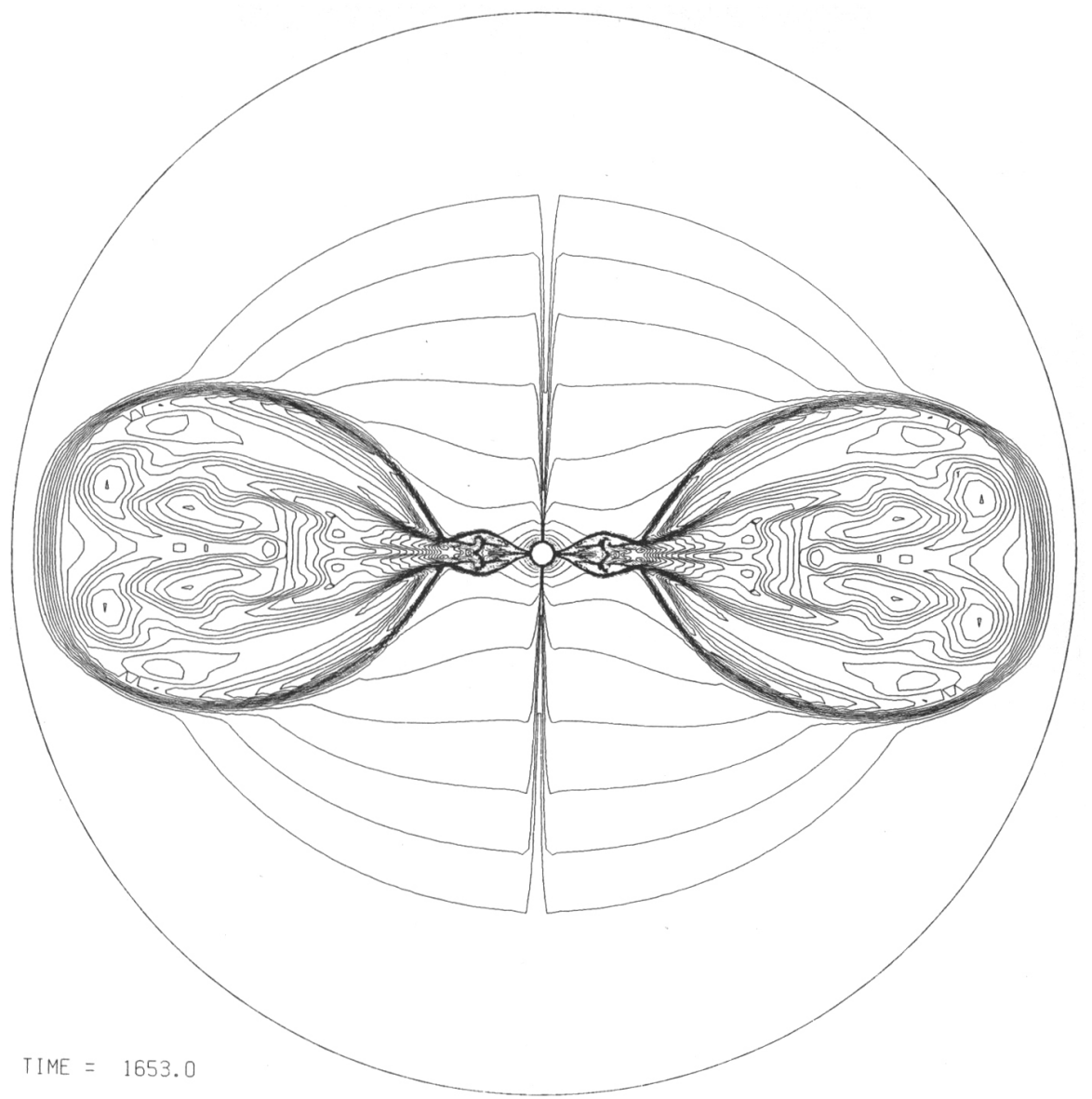

Figure 5a: Density contours showing torus formed by the high $l$ fluid in the $l=9 \sin ^{2}(\theta)$ model. Note location of shocks and the equatorial outflow in the torus

In the optically thick torus, how is the intense heat generated in the center transported out to the disk surface? What sort of wind will the near- or super-Eddington radiation flux produce?

A related issue is the question of jet acceleration from accretion disks and tori. The numerical models have shown how funnels naturally arise in inflows with angular momentum, and have demonstrated how outflows in the form of hollow jets could be produced by purely hydrodynamic processes. The scenarios and theoretical questions surrounding jet formation are reviewed by BLANDFORD in this volume.

One very significant aspect which is not included by these calculations are the nonaxisymmetric instabilities discovered by PAPALOIZOU and PRINGLE [35], [36]. Unlike the usual accretion disk instabilities which depend on the nature of the viscous heating and the radiative cooling, the instability arises from the three-dimensional hydrodynamic equations alone. GOLDREICH and NARAYAN [37] have suggested that these instabilities may be produced by waves amplified in the interior of a thick torus as they reflect off the corotation point in the interior. A portion of such a wave is transmitted through corotation but the energy in the transmitted wave has the opposite sign as that in the reflected wave. Reflection off the torus boundary permits the waves to be amplified repeatedly. It is possible that these instabilities may provide an effective minimum angular momentum transport, and that constant, or near constant angular momentum 


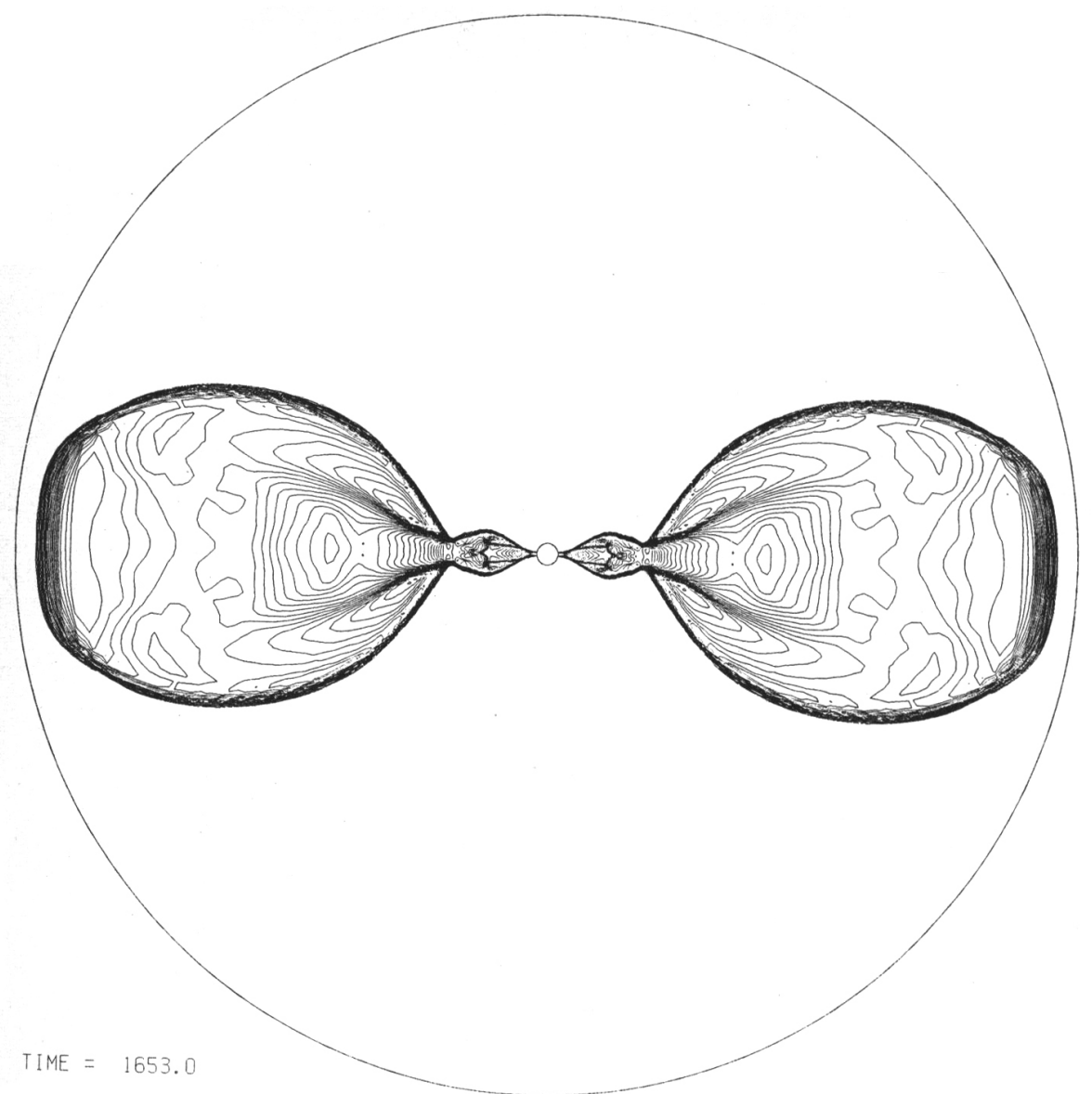

Figure 5b: Pressure contours for model shown in Fig. 5a

torl are not stable. This is currently a subject of active research.

I would like to close by suggesting that the evolution of central engine theory can be compared with the theory of stellar structure. Our theoretical understanding of stars began by considering them to be polytropic balls of gas of uniform composition, supported against their selfgravity by pressure forces. Now, stellar models using nuclear reaction networks, realistic opacities and a detailed equation of state are produced on computers. Such models have verifled or corrected many aspects of the original theories while providing the evolutionary tracks which are compared with observations. The central engine is the new stellar evolution problem. Accretion disks around black holes are (at least) two dimensional structures. Much of the basic physics is still not understood, e.g., the nature of the viscosity which generates heat in the disk is unknown. However, since the black hole provides the fixed gravitational potential it has been possible to create descriptions of simplified accretion structures and flows. Thus current theory is at the "polytrope" stage of development; we have the correct order of magnitude, some likely shapes and structures, but lack sufficient detail of the internal workings. Substantial theoretical progress is required, first to obtain the equations which describe the physics of the central engine, and then to develop the computational expertise to solve those equations.

I would like to thank the Max Planck Institute for its support, both at the I.A.U. Colloquium, and during the spring of 1984 when some of these numerical calculations were performed. Larry 
Smarr provided continual input over the course of this work, and his work is gratefully acknowledged, as are the contributions of colleagues too numerous to mention individually. Dimitri Mihalas and Karl-Heinz Winkler deserve credit for organizing a fine colloquium. This work was supported in part by NSF grants PHY-B511426 and AST-B213001, and by a Bantrell Fellowship at Caltech.

\section{References}

1. M. J. Rees: Phys. Scripta, 17, 193 (1978)

2. C. Misner, K. Thorne, and J. Wheeler: Gravitation (Freeman, San Francisco 1973)

3. M. Abramowicz, M. Jaroszynski, M. Sikora: Astron. Astrophs., 63, 221 (1978)

4. M. Kozlowski, M. Jaroszynski, M. Abramowicz: Astron. Astrophs., 63, 209 (1978)

5. D. Lynden-Bell, Phys. Scripta, 17, 185 (1978)

6. J. M. Bardeen, J. A. Petterson: Astrophys. Jour. Lett., 195, L65 (1975)

7. R. D. Blandford, R. L. Znajek: Mon. Not. Roy. Astr. Soc., 179. 433.

B. M. J. Rees, M. C. Begelman. R. D. Blandford, and E. S. Phinney: Nature, 295, 17 (1982)

9. M. C. Begelman, R. D. Blandford, M. J. Rees: Rev. Mod. Phys., 56, 255 (1984)

10. M. J. Rees: Ann. Rev. Astron. Astrophys., 22, 471 (1984)

11. S. Shapiro: Astrophys. Jour., 180, 531 (1973)

12. P. Meszaros: Astron. Astrophys., 44. 59 (1975)

13. M. C. Begelman: Mon. Not. Roy. Astr. Soc., 184. 53 (1978)

14. R. A. Flammang: Mon. Not. Roy. Astr. Soc., 199, 833 (1982)

15. M. C. Begelman: Mon. Not. Roy. Astr. Soc., 187, 237 (1979)

16. D. L. Gilden, J. C. Wheeler: Astrophys. Jour., 239, 705 (1980)

17. J. P. Ostriker, R. McCray, R. Weaver, and A. Yahil, Astrophys. Jour. Lett., 208, L61 (1976)

18. L. L. Cowie, J. P. Ostriker, A. A. Stark: Astrophys. Jour., 226, 1041 (1978)

19. N. I. Shakura, R. A. Sunyaev: Astron. Astrophys., 24, 337 (1973)

20. 1. Novikov, K. S. Thorne: "Astrophysics of Black Holes"; in Black Holes, eds. B. DeWitt and C. DeWitt, (Gordon and Breach, New York 1973), p. 343

21. J. E. Pringle: Ann. Rev. Astron. Astrophys., 19, 39 (1981)

22. M. Abramowicz, M. Jaroszynski, M. Sikora: Astron. Astrophys., 242, $772(1980)$

23. M. Abramowicz, T, Piran: Astrophys. Jour. Lett., 241, L7 (1980)

24. M. Sikora, D. B. Wilson: Mon. Not. Roy. Astr. Soc., 196, 529 (1981)

25. L. Nobili, R. Turolla, M. Calvani: Lett. al Nuovo Cimento, 35. 335 (1982)

26. T. Piran: Astrophs. Jour. Lett., 257, L23, (1982) 
27. M. Begelman, M. J. Rees: Mon. Not. Roy. Astr. Soc., 206, 204 (1984)

28. S. L. Shapiro, A. P. Lightman, D. M. Eardley: Astrophys. Jour., 204, 187 (1976)

29. E. P. T. Liang, R. H. Price: Astrophys. Jour., 21B. 247 (1977)

30. J. R. Wilson: Astrophys. Jour,, 173, 431 (1972)

31. J. F. Hawley, L. L. Smarr, J. R. Wilson: Astrophys. Jour., 277, 296 (1984)

32. J. F. Hawley, L. L. Smarr. J. R. Wilson: Astrophys. Jour. Suppl,, 55. $211(1984)$

33. J. F. Hawley, L. L. Smarr: "New Paradigms for Black Hole Accretion from High Resolution Supercomputer Experiments", in AIP Proceedings of the Los Alamos Workshop on Magnetospheric Phenomena in Astrophysics, ed. R. Epstein (AIP, New York 1985) in press

34. M. A. Abramowicz, and W. H. Zurek: Astrophys. Jour., 246, 314 (1981) 35. J. C. B. Papaloizou, J. E. Pringle: Mon. Not. Roy. Astr. Soc, 20B.

$721(1084)$

36. J. C. B. Papaloizou, J. E. Pringle: Mon. Not. Roy. Astr. Soc., 213, 799 (1985)

37. P. Goldreich, R. Narayan: Mon. Not. Roy. Astr. Soc,, 213, 7P (1985)

\section{Discussion Following Paper Presented by J. Hawley}

NORMAN: You stressed the importance of "inertial confinement" in determining how much matter is retained near the black hole in the form of a thick disk vs. how much escapes in the form of a hot wind or hollow jet. Could you explain why, in your "best confinement" case of thick inflow, the matter avoids the funnel wall as it flows in, thus providing an escape route for the shock heated gas? Could you imagine an even better confinement scenario?

HAWLEY: Confinement is produced by the thickness of the inflow; a purely equatorial flow permits the post-shock fluid to escape almost radially outward, above and below the equator. How thick an inflow will be depends on its temperature (the hotter, the thicker), and how its being fed into the system at large radius. Any inflow will avoid the funnel wall unless it is held against that wall by a strong pressure gradient; such a hot inflow would be a better confinement scenario.

SHULL: The Papaloizou-Pringle global, dynamic instability appeared to depend on reflection of waves off inner boundaries and amplification near the corotation radius. Could you see any evidence for this instability (particularly with an axisymmetric code)?

HAWLEY: The code I described here can not address the issue except to demonstrate the efficacy of the inner torus surface to reflect waves. Test calculations with a cylindrically symmetric $(r, \varphi)$ code have suggested perturbation growth. Accurate simulations will probably require a full three-dimensional treatment.

MICHEL: What does specifically assuming the central object to be a black hole buy you insofar as explaining quasars? The "funnel" for example, is a Newtonian effect appropriate for a thick disk orbiting any absorbing object.

HAWLEY: The compactness of the emission region, and the huge energy output tend to constrain models without black holes to be rather contrived. The funnel is a Newtonian effect insofar as it represents the presence of a centrifugal barrier keeping flows with angular momentum away from the orbital axis. The relativistic effect results from the "hole" at the bottom; material in the funnel must either fall in or fow out, but cannot be stationary. 
FISHER: Is it true that in your code there is no source or sink term in the energy equation other than viscous dissipation by shocks? If so, do you think that the inclusion of radiative cooling would affect the structure of your accretion shocks and flows?

HAWLEY: There are no other sources or sinks in the code. In my accretion flows, everything takes place on a treefall timescale, and emission mechanisms which also take place on that timescale (e.g. electron cooling) could have a very significant effect. In any event, the evolution of a torus will depend almost entirely on multi-dimensional radiative/viscous effects which are very difficult to model numerically, even if we had a complete theory of such effects.

SCHNEIDER: How did you estimate the accretion efficiencies you talked about?

HAWLEY: In these inflow models, the "efficiency" is not a true accretion efficiency unless the inflow is steady state. Otherwise it simply reflects the net release of gravitational binding energy in producing the thick disk.

OPHER: How would thermonuclear reactions modify your scenarios, in particular in the stagnation regions where you have high density and temperature?

HAWLEY: The energy released by nuclear reactions would probably not significantly add to the net luminosity, but nevertheless have important dynamic effects, e.g. creating entropy gradients, instabilities, etc. 